\title{
Platz zum Denken!? - Praxis und Reflexion als entscheidende Impulse in der brasilianischen Deutschlehrerausbildung
}

[A place to think!? - Practical experience, reflection and its core influence in Training for German Teachers in Brazil]

http://dx.doi.org/10.11606/1982-88372236178

\author{
Ynaiara dos Santos ${ }^{1}$ \\ Hudson Kleinbing ${ }^{2}$ \\ Liane Scribelk ${ }^{3}$ \\ Paul Voerkel ${ }^{4}$
}

\begin{abstract}
With reference to the undergraduate course on Portuguese and German at a Federal University in Southeast Brazil, the article first describes some fundamental elements of German language teacher training in the country. Hereby it becomes clear how important the integration of theory and practice is for the training of student's competencies. In this context, the article secondly gives insights to a subject offered at that university, in which the participants use bases of action research by reflecting on their own teaching and thus strengthening their professional performance as teachers. Finally, some suggestions from this experience will be formulated for a meaningful integration of reflective elements in the teacher training.
\end{abstract}

Keywords: Brazil; Training for German Teachers; Action Research.

Zusammenfassung: Unter Bezugnahme auf den Deutschstudiengang an einer Bundesuniversität im Südosten Brasiliens beschreibt der Artikel zunächst einige Grundlagen der Deutschlehrerausbildung des Landes. Dabei wird deutlich, wie wichtig die Verknüpfung von Theorie und Praxis für die Kompetenzentwicklung der Studierenden ist. Vor diesem Hintergrund wird ein Seminarangebot aus dem Deutschstudiengang der Universität vorgestellt, bei dem die Teilnehmenden im Rahmen der Aktionsforschung ihren eigenen Unterricht reflektieren und damit ihr professionelles Handeln als Lehrkräfte stärken können. Aus den Erfahrungen heraus werden

\footnotetext{
${ }^{1}$ Universidade Federal do Rio de Janeiro, Av. Horácio Macedo, 2151, Rio de Janeiro, RJ, Brasil, 21941 917, Brasil. E-Mail: yaya_cmp@hotmail.com

${ }^{2}$ Universidade Federal do Rio de Janeiro, Av. Horácio Macedo, 2151, Rio de Janeiro, RJ, Brasil, 21941917, Brasil. E-Mail: hudson.kleinbing@ hotmail.com

${ }^{3}$ Universidade Federal do Rio de Janeiro, Av. Horácio Macedo, 2151, Rio de Janeiro, RJ, Brasil, 21941 917, Brasil. E-Mail: liane.scribelk@ hotmail.com

${ }^{4}$ Universidade Federal do Rio de Janeiro, Av. Horácio Macedo, 2151, Rio de Janeiro, RJ, Brasil, 21941 917, Brasil. E-Mail: paul.voerkel@gmail.com
}

(cc) BY-NC

Pandaemonium, São Paulo, v. 22, n. 36, jan.-abr. 2019, p. 178-206 
SANTOS, Y.; KLEINBING, H.; SCRIBELK, L.; VOERKEL, P. - Platz zum Denken!?

schließlich einige Vorschläge für eine sinnvolle Integration reflexiver Elemente in die Lehrerausbildung formuliert.

Stichwörter: Brasilien; Deutschlehrerausbildung; Aktionsforschung.

\section{Einführung}

Seit langem schon beschäftigen sich Menschen mit der Vermittlung von Fremdsprachen, um zu verstehen, wie Lernen grundsätzlich funktioniert und wie es weiter verbessert werden kann. Systematisch erforscht werden die dem Fremdspracherwerb zugrundeliegenden Prozesse jedoch erst seit Ende des 19. Jahrhunderts mit der Etablierung der Sprachwissenschaften und der wissenschaftlichen Pädagogik (vgl. EDMONDSON; HOUSE 2011: 15).

Im deutschsprachigen Raum haben sich in den 1960er und 1970er Jahren aus der Beschäftigung mit dem Lehren und Lernen von Fremdsprachen mehrere wissenschaftlich begründete Fachgebiete entwickelt, in erster Linie die Sprachlehr- und -lernforschung und die Fremdsprachendidaktik (vgl. EDMONDSON; HOUSE 2011: 2f., 16f.; GOER; KÖLLER 2016: 21; KÖNIGS 2017: 322). Aus den vielfältigen Forschungsergebnissen der Fachrichtung lassen sich dabei drei besonders relevante Aussagen herausgreifen. Der Erwerb von Fremdsprachen ist erstens ein hoch komplexer und multifaktorieller Prozess, der von verschiedenen Variablen beeinflusst wird (vgl. BALLWEG et al. 2013: 38; FELDMEIER 2014: 255; RIEMER 2017: 320f.). Aus der Vielfalt der Faktoren mit Einfluss auf das Fremdsprachenlernen ergibt sich zweitens die Erkenntnis, dass eine einzelne Lerntheorie (ebenso wie eine einzelne Sprachlernmethode) den Lernprozess nicht sinnvoll abbilden kann (vgl. u. a. BALLWEG et al. 2013: 63). Folge dieser Erkenntnisse ist die Überwindung von „Makromethoden“ zum Fremdsprachenlernen (die sogenannte „Postmethoden-Ära“, vgl. DoFF 2016: 321) und ein zunehmender Eingang von lerntheoretisch fundierten Vorschlägen in die Theorie und Praxis des Fremdsprachenlernens. Drittens schließlich begründet die Komplexität von Sprachlehrund -lernprozessen die Notwendigkeit zur interdisziplinären Zusammenarbeit mit Nachbardisziplinen, wie etwa der Linguistik und der Psychologie (vgl. EDMONDSON; HOUSE 2011: 18).

Die Komplexität des Fremdsprachenlernens zeigt, wie groß die Herausforderungen bei der Ausbildung von Fremdsprachenlehrkräften sind. Entsprechende Studiengänge haben vor allem die Aufgabe, Studierende auf ihre 
SANTOS, Y.; KLEINBING, H.; SCRIBELK, L.; VOERKEL, P. - Platz zum Denken!?

zukünftige Tätigkeit als Lehrpersonen vorzubereiten. Dies erfordert, neben der Vermittlung solider fachlicher Kenntnisse, noch eine Reihe anderer Voraussetzungen, wie etwa: Zeit, Förderung und Unterrichtspraxis. Aus dieser Gemengelage haben sich in den letzten Jahren eine Reihe von Prinzipien und Standards für die Lehrerausbildung entwickelt, die im Sinne einer Professionalisierung des Lehrberufs international diskutiert und inzwischen auch weitgehend anerkannt werden (vgl. MOHR; SCHART 2016: 299f., VOERKEL 2017a: 60-69, 268-274). Eine Einbeziehung dieser Prinzipien in die Ausbildung zukünftiger Lehrkräfte kommt allerdings weltweit erst Stück für Stück in Gang.

In Hinblick auf die Lehrerausbildung steht auch Brasilien vor umfassenden Aufgaben. Einige der wesentlichen Herausforderungen werden in diesem Beitrag aus der Innenperspektive beschrieben: drei der vier Verfasser dieses Artikels sind Studierende einer öffentlichen brasilianischen Bundesuniversität und befinden sich in der letzten Phase des Lehramtsstudiums Deutsch-Portugiesisch. Ziel dieses Artikels ist es dabei nicht, Kritik an der Struktur und den Inhalten des Lehramtsstudiums zu üben, sondern anhand eines konkreten Beispiels aufzuzeigen, dass aktuelle Erkenntnisse der Fremdsprachendidaktik im Rahmen der vorhandenen Möglichkeiten in die bereits bestehenden Studiengänge integriert werden können (vgl. MOHR; SCHART 2016: 294). Der damit angestrebte Beitrag zur Professionalisierung und Entwicklung der Lehrerbildung kann sowohl die Studierenden, die Lehrenden als auch das Fach selbst positiv beeinflussen.

\section{Zur Lehrerausbildung in Brasilien und ihren Herausforderungen}

Ein Hochschulstudium mit Lehramtsabschluss ist in Brasilien erst seit dem Jahr 2000 eine Grundvoraussetzung, um sich auf eine Stelle als Lehrkraft an Elementarschulen bewerben zu können (vgl. PUPP SPINASSÉ 2005: 118). Für die schulische Mittel- und Oberstufe gilt die entsprechende Verpflichtung jedoch bereits wesentlich länger: Seit den 1940er Jahren wurden Lehramtsstudiengänge für diese Zielgruppe im Rahmen der universitären LetrasStudiengänge eingerichtet, wobei in der Regel ein einzelnes Schulfach für die Dauer von vier Jahren studiert wird (vgl. RICHTER 2013: 185).

Das Lehramtsstudium für Deutsch wird in Brasilien jedoch üblicherweise in Kombination mit Portugiesisch angeboten: Lediglich an drei von 17 Universitäten mit Pandaemonium, São Paulo, v. 22, n. 36, jan.-abr. 2019, p. 178-206 
SANTOS, Y.; KLEINBING, H.; SCRIBELK, L.; VOERKEL, P. - Platz zum Denken!?

Deutschstudiengängen besteht Deutsch als Einzelstudium, an den anderen Hochschulen ist es entweder verpflichtend oder empfohlen, einen Doppelabschluss zu erwerben (vgl. VOERKEL 2018). In der Konsequenz bedeutet diese Fächerkombination ein breites Spektrum verschiedener Lehrangebote bzw. Pflichtfächer: Neben dem Erwerb der Fremdsprache, die einen Schwerpunkt an allen Studienstandorten darstellt (vgl. VOERKEL 2017a: 285-291), gibt es obligatorisch zu belegende Seminare in brasilianischer, portugiesischer und deutscher Literatur, portugiesischer Sprachwissenschaft, Didaktik und brasilianischer Bildung. Mit Ausnahme der Sprachkurse haben alle Lehrveranstaltungen eine Gemeinsamkeit: Sie sind - übrigens auch dort, wo es durchaus Anknüpfungspunkte gäbe, wie z. B. in Literaturseminaren - stark von der Theorievermittlung geprägt und nehmen kaum Bezug auf die berufliche Praxis nach dem Studium.

Das Fehlen der Praxiskomponente ist vor allem deswegen bedenklich, weil die Mehrheit der Letras-Studenten nach ihrem Abschluss als Lehrkräfte tätig ist. Nach Einschätzung der Faculdade de Letras an der Bundesuniversität UFRJ arbeitet ein Großteil der Absolventen als Lehrer, und eine breit angelegte Studie über den Verbleib von Absolventen brasilianischer Deutschstudiengänge kommt auf einen Anteil von bis zu 90\% in der Bildung engagierten Alumni (vgl. VOERKEL 2017a: 339). Die Erkenntnis, dass deutlich mehr Letras-Absolventen letztendlich als Lehrer arbeiten als üblicherweise angenommen (vgl. VOERKEL 2017b), führt erst langsam zu einem Umdenken an den Hochschulen, was sich u. a. in den Diskussionen um neue Curricula äußert. Bisher lässt sich jedoch feststellen, dass das Lehramtsstudium nach wie vor stark theoriebetont ist und wesentliche Ausbildungsmerkmale für die Praxis fehlen.

Dabei ist es nicht so, dass das Bewusstsein für die hohe Relevanz von Praxiserfahrungen in der Lehramtsausbildung fehlen würde. Zur Umsetzung des 1996 verabschiedeten Bildungsrahmenplans (im Original: Lei de Diretrizes e Bases - LDB) wurde Anfang der 2000er Jahre in mehreren Gesetzesvorlagen die Struktur der Lehramtsstudiengänge festgeschrieben, u. a. mit der Mindestanforderungen von 400 Stunden begleiteter Praktika an (öffentlichen) Schulen (vgl. STANKE 2014: 43). Allerdings scheitert der hohe Anspruch im Fall von Deutsch schlicht an einem Mangel an Möglichkeiten: Von über 50.000.000 Schülerinnen und Schülern im Land lernen weniger als 100.000 Deutsch - was einem Prozentwert von kaum 0,2\% entspricht (vgl. AuswÄrtiges Amt 2015: 9). Sind Schulen mit Deutschangebot in den beiden südlichen

Pandaemonium, São Paulo, v. 22, n. 36, jan.-abr. 2019, p. 178-206 
SANTOS, Y.; KLEINBING, H.; SCRIBELK, L.; VOERKEL, P. - Platz zum Denken!?

Bundesländern Rio Grande do Sul und Santa Catarina noch recht weit verbreitet ${ }^{5}$, gibt es in den anderen Landesteilen sehr viel weniger Angebote. Dies gilt auch für die Metropolregion Rio de Janeiro: zwar hat sich der Arbeitsmarkt für Deutschlehrkräfte dort in den letzten 20 Jahren dynamisch entwickelt, und neben mehreren Sprachinstituten bieten allein vier Privatschulen Unterricht in Deutsch als Fremdsprache an (vgl. STANKE; BOLACIO 2014: 127). Curricular verankert war ein Deutschangebot aber bis Anfang 2018 an keiner öffentlichen Schule, während gleichzeitig drei staatliche Universitäten in Lehramtsstudiengängen Deutschlehrkräfte ausbilden.

Das weitgehende Fehlen von schulischem Deutschunterricht an öffentlichen Einrichtungen hat zur Folge, dass viele Lehramtsstudierende ihr Praktikum nicht in der vorgesehenen Weise absolvieren können. Sie empfinden das als großen Mangel, ist ein Praktikum doch eine optimale Möglichkeit, während des Studiums erste Lehrerfahrungen zu sammeln und die Realität des Deutschunterrichts im schulischen Kontext kennenzulernen (vgl. ZYDATISS 1996). Eine Folge der fehlenden Anwendungsmöglichkeiten ist eine sinkende Motivation, denn die Studierenden möchten nicht nur die „großen Theorien“ kennenlernen, sondern auch selbst aktiv werden.

Im Fall der Bundesuniversität UFRJ können Studierende eine Lösung für die begrenzten Praktikumsmöglichkeiten innerhalb der eigenen Institution finden, und zwar im Sprachlernzentrum namens CLAC, an dem das Angebot von Deutsch als Fremdsprache-Kursen für Jugendliche und Erwachsene besteht. Hier können die LetrasStudierenden ein bezahltes Praktikum absolvieren, währenddessen sie von Dozenten begleitet und beraten werden, Ideen mit Kommilitonen austauschen, ausprobieren, inwieweit die erlernten Theorien in der Praxis funktionieren und auch erste Erfahrungen und Routinen im Unterrichten sammeln. Zudem ist die Tätigkeit dort ein wichtiger Impuls, um zu reflektieren, welche Lehrstrategien sie in ihrem Unterricht bei CLAC (und in der Zukunft auch in anderen Unterrichtssituationen) auswählen und benutzen können und sollen. Insbesondere die Möglichkeit des Nachdenkens über die Unterrichtsprozesse erscheint den Studierenden wichtig für ihre Ausbildung und Professionalisierung, was sich auch in den Aussagen von Kommilitonen aus dem Deutschbereich wiederfindet:

\footnotetext{
${ }^{5}$ Die stärkere Präsenz der deutschen Sprache und Kultur im Süden Brasiliens hat vor allem historische Gründe (vgl. VOERKEL 2017a: 17-20). Die Folgen wirken bis heute: Von den reichlich 300 Schulen mit Deutschangebot im Land entfallen mehr als $2 / 3$ auf die beiden südlichen Bundesländer (vgl. VOERKEL 2017a: 53).
}

Pandaemonium, São Paulo, v. 22, n. 36, jan.-abr. 2019, p. 178-206 
SANTOS, Y.; KLEINBING, H.; SCRIBELK, L.; VOERKEL, P. - Platz zum Denken!?

„Wir glauben, dass dieses Nachdenken die Erfahrung unserer Ausbildung wertvoller macht.“

Mit dem Sprachlernzentrum ergibt sich - zumindest für einen Teil der Studierenden - die Möglichkeit zur Verzahnung von Theorie und Praxis. Da dies für sie einen hohen Stellenwert besitzt, wird das Sprachlernzentrum der UFRJ und sein Potential im folgenden Abschnitt genauer beschrieben.

\section{Das Sprachlernzentrum der UFRJ: CLAC}

Das Sprachlernzentrum CLAC ${ }^{6}$ an der Bundesuniversität von Rio de Janeiro (UFRJ) nahm 1988 seine Tätigkeit auf und startete anfangs mit sechs Fremdsprachen. Heute können die Interessenten aus einem breiten Angebot von 24 Sprachkursen mit verschiedenen Schwerpunkten auswählen ${ }^{7}$. Seit seiner Gründung hat das CLAC viele Veränderungen durchlaufen, insbesondere in Hinblick auf die Erweiterung des Kursangebots. Dennoch blieb die ursprüngliche Zielsetzung erhalten, nämlich einen produktiven und sinnvollen Austausch zwischen der Universität und der Gesellschaft zu fördern. Dies geschieht durch die Verankerung in beiden Kontexten: Das CLAC ist als Projeto de Extensão ${ }^{8}$ direkt an der Letras-Abteilung der Universität angesiedelt und wird von der Hochschule verwaltet, gleichzeitig steht es aber sowohl den Universitätsangehörigen als auch Lernern von außerhalb der Hochschule offen.

Das Sprachlernzentrum verfolgt dabei nicht nur das Ziel, die Grenzen der Universität zu überwinden, sondern auch ein Praxisangebot für die Lehrenden in Ausbildung bereitzustellen. Viele Studierende - insbesondere der „kleineren Sprachen“ - haben keine Gelegenheit, die von ihnen studierte Philologie außerhalb des universitären Kontexts zu unterrichten. Deshalb wird das CLAC als ein wichtiges Instrument im

\footnotetext{
${ }^{6}$ Akronym für Centro de Línguas Aberto à Comunidade. Eine ungefähre Übersetzung ins Deutsche wäre: Sprachenzentrum für die Gemeinschaft.

${ }^{7}$ Konkret handelt es sich um folgende Angebote: Arabisch, Arabisch mit Schwerpunkt Sprechen, Deutsch, Deutsch mit Schwerpunkt Sprechen, Englisch, Englisch mit Schwerpunkt Sprechen, Englisch mit Schwerpunkt Lesen, Englisch für Lehrende, Französisch, Französisch mit Schwerpunkt Sprechen, Griechisch, Hebräisch, Italienisch, Italienisch mit Schwerpunkt Sprechen, Japanisch, Japanisch mit Schwerpunkt Sprechen, Latein, Libras (Brasilianische Gebärdensprache), Portugiesisch, Portugiesisch mit Schwerpunkt Grammatik, Portugiesisch mit Schwerpunkt Schreiben, Russisch und Spanisch.

${ }^{8}$ Die Extensão stellt neben der Lehre und der Forschung den dritten Schwerpunkt an brasilianischen Universitäten dar. Mit diesen außercurricularen Projekten sollen die Hochschulen direkt in die Gesellschaft hineinwirken (weitere Informationen dazu u. a. bei VOERKEL 2017a: 211f.).
}

Pandaemonium, São Paulo, v. 22, n. 36, jan.-abr. 2019, p. 178-206 
SANTOS, Y.; KLEINBING, H.; SCRIBELK, L.; VOERKEL, P. - Platz zum Denken!?

Studium betrachtet, das seine Bedeutung neben dem Sprachenangebot dezidiert durch die Betreuung der angehenden Lehrkräfte durch Universitätsdozenten und das damit verbundene akademische Profil gewinnt. Laut Zyngier und Liberali (2000) zählt zu den spezifischen Zielen des CLAC, reflektierende Lehrkräfte auszubilden, die nicht nur passend im Arbeitsmarkt platziert werden können, sondern auch in der Lage sind, kritisch und aufmerksam auf pädagogische Herausforderungen zu reagieren. So sollen sie beispielsweise die von ihnen verwendeten Materialien sorgfältig auswählen und weiterentwickeln, damit die Bedürfnisse und Bedarfe der Lerner erfüllt werden.

Schon allein aus Kapazitätsgründen können nicht alle, die an der Bundesuniversität UFRJ Deutsch studieren, im CLAC unterrichten. Aus diesem Grund wurden konkrete Anforderungen erarbeitet, welche die zukünftigen CLAC-Lehrkräfte erfüllen müssen: mindestens vier Semester Deutschstudium an der Universität und ein guter Notendurchschnitt (gefordert wird ein Minimum von 8/10 Punkten in Deutsch und 7/10 Punkten in den anderen Fächern). Zudem müssen alle interessierten Lehrenden eine Eingangsprüfung bestehen, die sich aus einer schriftlichen Prüfung und einem Interview auf Deutsch zusammensetzt. Bewerber für Sprachkurse in Englisch und Spanisch müssen im CLAC zudem eine Lehrprobe ablegen, was allerding für Deutsch nicht notwendig ist. Gleichwohl durchlaufen die ausgewählten Kandidaten ein kurzes Training, das von den erfahreneren Lehrenden geleitet wird. Nach dieser Anleitung dürfen die Teilnehmer dann unterrichten, wobei zu Beginn der Lehrtätigkeit nur ein Kurs unterrichtet wird. Später kann die Zahl der Sprachkursgruppen bis auf drei steigen.

Die Lehramtsstudierenden im CLAC müssen einmal pro Woche ein pädagogisches Betreuungsangebot wahrnehmen. Diese Treffen werden von den Professoren bzw. Dozenten des Instituts vorbereitet und behandeln bestimmte Schwerpunktthemen. Sie bieten dabei einen Raum, in dem die Lehrenden sowohl ihre Fragen stellen können als auch ihre Erfahrung als Lehrer teilen. Für die professionelle Weiterentwicklung produktiv sind auch die Vorträge, die alle Lehrenden in diesem Rahmen halten müssen. In den Vorträgen haben sie die Chance, einen bestimmten Aspekt der Lehrtätigkeit oder eine bestimmte Lehr- und Übungsform vorzustellen und sich darüber auszutauschen. Der Vortrag und die Teilnahme an den Beratungen werden von den Dozenten evaluiert - sie werden deswegen sowohl aus inhaltlichen als auch aus formellen Gründen von den Teilnehmenden als sehr wichtig empfunden. 
SANTOS, Y.; KLEINBING, H.; SCRIBELK, L.; VOERKEL, P. - Platz zum Denken!?

Zweifelsohne ist der für die weitere Lehrtätigkeit prägendste Aspekt der gesamten CLAC-Erfahrung die Freiheit als Lehrer. Zwar ist vorgeschrieben, ein kurstragendes Lehrwerk zu benutzen (im Fall von Deutsch das Buch „Menschen“ aus dem HueberVerlag), aber die Lehramtsstudenten können autonom entscheiden, wie sie mit dem Lehrwerk arbeiten möchten. Diese didaktische Freiheit und die wöchentlichen Beratungen bilden aus Sicht der Lehramtsstudierenden das Herzstück der CLACTätigkeit.

Bei allen schon bestehenden Möglichkeiten ist anzumerken, dass ein noch stärkerer Fokus auf die Selbstreflexion wünschenswert wäre. Da der Anspruch des CLAC grundsätzlich in der Förderung der kritischen Bildung liegt, ergibt sich hier großes Potenzial. Von Seiten der Studierenden wird einerseits angeregt, bei der Betreuung einen stärkeren Impuls zur Reflexion zu erhalten, und andererseits noch mehr bei der Planung und Durchführung von Projekten unterstützt zu werden - sowohl in Hinblick auf die Unterrichtspraxis als auch auf die fachlich-wissenschaftliche Aus- und Weiterbildung. Diese Maßnahmen könnten nicht nur die Autonomie und die Motivation der Lehrenden stärken, sondern auch die Nachhaltigkeit des Lernprozesses und die Qualität der Reflexion im CLAC.

\section{Zur theoretischen Rahmensetzung: Aktionsforschung}

Für die zukünftigen Deutschlehrer ist es von großer Bedeutung, bereits während ihres Studiums die Lehrpraxis im Blick zu haben. Es ist dabei wichtig, einen passenden Raum zu finden, um die zukünftigen Tätigkeiten zu reflektieren. Diese Möglichkeit besteht vor allem dann, wenn begleitete Praktika im Rahmen der Lehrerbildung im Curriculum verankert sind und durch die Lehrkräfte der Fachdidaktiken auch wirklich angeboten werden. Begründungen für die Relevanz einer solchen Form von Erfahrungen finden sich in der Fachliteratur an zahlreichen Stellen, so z. B.:

Reflexion ist eine Form von Lernen aus Erfahrung. Sie bedeutet konstruktive Verarbeitung von Erfahrungen. Da auf einmalige, ungewisse und widersprüchliche Situationen nicht in dem Sinne vorbereitet werden kann, dass sich vorweg bestimmen ließe, wie in solchen Situationen zu handeln ist, ist die Fähigkeit zur reflexiven Verarbeitung von Erfahrungen eine wesentliche Komponente der professionellen Kompetenz von Lehrpersonen. (HERZOG 1995: 271) 
SANTOS, Y.; KLEINBING, H.; SCRIBELK, L.; VOERKEL, P. - Platz zum Denken!?

Die begleitete Reflexion sorgt dafür, dass die angehenden Lehrkräfte über ihre Unterrichtspraxis nachdenken und sich selbst entdecken, sie sind damit ein wichtiges Instrument für die Professionalisierung des Lehrerhandelns (vgl. BENITT 2015: 65). Wichtig für den Erfolg ist dabei, dass die Reflexion einer gewissen Steuerung und vor allem Struktur unterliegt (vgl. WALlace 2010: 14f.). Dazu liegt ein in diesem Sinne handhabbares und inzwischen bewährtes Instrument mit der Aktionsforschung vor. Dieses Konzept - auch bekannt als Lehrerforschung oder unter anderen verwandten Termini (vgl. SCHART; LEGUTKE 2012: 186f.; MOHR; SCHART 2016: 292, ABENDROTHTIMMER 2017: 196f.) - existiert bereits seit den 1940er Jahren unter dem Begriff der Action Research, hat sich im deutschen Sprachraum aber erst seit etwa zehn Jahren verstärkt durchgesetzt (vgl. BENITT 2015: 66ff.). Bedeutsam für diesen Durchbruch war u. a. die viel rezipierte Publikation „Lehrerinnen und Lehrer erforschen ihren Unterricht“, veröffentlicht von Altrichter und Posch im Jahr 2007. Weitere Verbreitung in der Praxis fand das Konzept ab 2012 mit den Materialien der aktuellen Fortbildungsreihe „Deutsch Lehren Lernen“ (DLL), die vom Goethe-Institut in Zusammenarbeit mit mehreren im Fachbereich aktiven Universitäten entwickelt worden ist (vgl. MOHR; SCHART 2016: 291). Die Grundideen der Aktionsforschung können dabei folgendermaßen zusammengefasst werden:

Aktionsforschung [...] ist eine Art von Forschung, die von professionell Handelnden (z. B. Lehrenden) direkt in ihrem Praxisumfeld durchgeführt wird. Ziel ist, eine Intervention zur Verbesserung der Praxis - die „Aktion“ - forschend zu begleiten (Burns 2010: 1). Dabei entsteht ein Kreislauf von Aktion, Forschung und Reflexion, der mehrfach durchlaufen werden kann und sowohl einen Erkenntnisfortschritt in Hinblick auf die Theorie als auch einen Entwicklungsfortschritt in Hinblick auf die Praxis erbringt (Altrichter \& Posch 2007: 27) und aus diesem Grund oft auch als Spirale beschrieben wird (Herr \& Anderson 2015: 5). Vom alltäglichen Praxishandeln - das ja oftmals auch Phasen des Ausprobierens, Beobachtens und Reflektierens enthält - unterscheidet sich Aktionsforschung durch ihre Systematik (Riemer 2010: 361), die Dokumentation des Prozesses (Feldmeier 2014: 261) und die Verbreitung der Ergebnisse (Altricher \& Posch 2007: 29). (BOECKMANN 2016: 592)

Wenn Lehrkräfte auf Grundlage ihrer eigenen Unterrichtspraxis zum Nachdenken angeregt werden und sich bewusst mit der Aktionsforschung auseinandersetzen, gibt ihnen dies die Möglichkeit, mehr Autonomie zu gewinnen und sich in ihrer eigenen Lehrpraxis weiterzuentwickeln. Dabei ist festzuhalten, dass das Verfahren nicht für jeden Kontext uneingeschränkt zu empfehlen ist und sicher auch nicht jede Lehrkraft mit dem Vorgehen der Aktionsforschung zurechtkommt (vgl. BENITT 2015: 74ff.). In jedem Fall sollte die Motivation am Anfang stehen, auf das eigene Unterrichten Einfluss zu nehmen, 
SANTOS, Y.; KLEINBING, H.; SCRIBELK, L.; VOERKEL, P. - Platz zum Denken!?

weil im Zentrum der Bemühungen letztendlich immer der Wunsch steht, den eigenen Unterricht zu verbessern (vgl. FELDMEIER 2014: 255).

Auch in Brasilien gewinnt die Aktionsforschung zunehmend an Bedeutung, hier unter dem Begriff der Pesquisa-Ação. Laut Franco-Santoro (2005) lassen sich dabei vor allem drei Formen unterscheiden:

a) Der kollaborative Ansatz, bei dem der Forscher in seiner Rolle als teilnehmender Akteur vor allem auf einen Impuls von Seiten der Lerner reagiert.

b) Der kritische Ansatz, bei dem es dem Forscher vor allem darum geht, die Machtverhältnisse zwischen Lehrer und Lerner sowie ihre jeweilige Rolle zu hinterfragen und sie in einem gemeinsamen Prozess zu verändern.

c) Der strategische Ansatz, bei dem der Impuls für die Veränderung vom Lehrer ausgeht und die teilnehmende Forschung auch weitgehend von ihm (ohne aktive Beteiligung der Lerner) geplant und durchgeführt wird.

Wie aus der Konzeptionierung und den Erfahrungen der DLL-Reihe deutlich wird, lassen sich die einzelnen Beweggründe und Vorgehensweisen nicht immer klar voneinander trennen und überlappen sich teilweise. In jedem Fall wird jedoch klar, dass die Rolle des Lehrers bei Veränderungen des Unterrichts nicht zu unterschätzen ist, insbesondere, weil sie für das gesamte Berufsleben gilt (vgl. WaLlaCE 2010, 4). Dementsprechend werden auch in der Fachliteratur diejenigen Kompetenzen, die wichtig für die persönliche und professionelle Entwicklung von Lehrpersonen sind, als hoch eingeschätzt: so liegen etwa die Fähigkeit zur Formativen Evaluation oder Rückmeldung/Feedback unter den Top-10 der relevantesten Punkte für Lernerfolg in der vielzitierten Meta-Studie von John Hattie (vgl. ZIERER 2016: 64-71). Daraus ergibt sich letztendlich die hohe Relevanz, die Reflexionsfähigkeit von Lehrpersonen bereits in der Ausbildung aktiv zu trainieren.

In Brasilien haben diese Erkenntnisse durchaus ihren Niederschlag gefunden, vor allem im Programa Institucional de Bolsas de Iniciação à Docência (PIBID), einem Lehrstipendium für die studienbegleitende Unterrichtserfahrung. Die Studierenden bekommen über das PIBID die Möglichkeit, im Sinne der Aktionsforschung ihre ersten Unterrichtserfahrungen in ihrer Rolle als Lehrkraft zu reflektieren und gleichzeitig eine konstruktive, kollegiale Rückmeldung zu erhalten. Sie können sich dabei während eines Jahres als Lehrkraft an öffentlichen Schulen ausprobieren und ihr didaktisches Handeln trainieren: zunächst über eine Hospitationsphase, und dann durch die zunehmende Pandaemonium, São Paulo, v. 22, n. 36, jan.-abr. 2019, p. 178-206 
SANTOS, Y.; KLEINBING, H.; SCRIBELK, L.; VOERKEL, P. - Platz zum Denken!?

Übernahme von Unterrichtssequenzen, meist in Form von Team-Teaching. Neben der Lehrtätigkeit werden vier Stunden pro Woche für die gemeinsame Vorbereitung des Unterrichts und die Auswertung der Erfahrungen in der Seminargruppe genutzt. Organisiert wird das Programm einerseits von Seiten der Universitäten (dort entweder von den jeweiligen Fachdidaktiken oder der Fakultät für Erziehungswissenschaften) und andererseits von den beteiligten Schulen, an denen der jeweilige Fachlehrer die Koordination übernimmt. Das Lehrstipendium selbst wird von der Coordenação de Aperfeiçoamento de Pessoal de Nivel Superior (Capes) ausgezahlt (vgl. PIBID 2018).

Die Aktivitäten im Rahmen des PIBID-Programms stellen eine Schnittstelle zwischen Schulen und Universitäten dar und können damit als sinnvolle Umsetzung wissenschaftlicher Erkenntnisse für die Praxis gesehen werden - zudem haben sie den Effekt, die Studierenden nachhaltig zu motivieren (vgl. VOERKEL 2017a, 301). Trotz dieser Erfolgsgeschichte kam es in den vergangen zwei Jahren $\mathrm{zu}$ massiven Budgetkürzungen in dem Programm, sodass sein Weiterbestehen keineswegs gesichert ist. Bereits heute haben längst nicht alle Interessenten die Möglichkeit, am PIBID teilzunehmen, weswegen es für die Fachdidaktiken umso wichtiger ist, den Studierenden sinnvolle Angebote zu unterbreiten, bei denen Theorie und Praxis verbunden werden und die Reflexion über das eigene Handeln im Unterricht bewusst gefördert wird.

\section{Methodisches Vorgehen}

Den Überlegungen zur Relevanz der Verknüpfung von Theorie und Praxis im Sinne der Aktionsforschung folgt auch das Setting der Studie, die von den Autoren dieses Artikels durchgeführt wurde. Diese wird auf den folgenden Seiten vorgestellt, wobei zunächst eine Beschreibung der institutionellen Einbettung in den Rahmen des Deutschstudiums an der Bundesuniversität UFRJ erfolgt.

Um das Lehramtsstudium abzuschließen, müssen die Studierenden laut Vorgabe des brasilianischen Bildungsministeriums 400 Stunden an Hospitationen und Unterrichtspraktika absolvieren. In Vorbereitung darauf sind einige Lehrveranstaltungen aus dem Didaktik-Bereich zu belegen. Dabei handelt es sich um zwei Semester Didaktik der Fremdsprache (im vorliegenden Fall: Deutsch) und zwei Semester Didaktik der Muttersprache (Portugiesisch). Grundlage für die folgenden Überlegungen sind die Erfahrungen aus einer Lehrveranstaltung für Didaktik der Fremdsprache, dem Fach

Pandaemonium, São Paulo, v. 22, n. 36, jan.-abr. 2019, p. 178-206 
SANTOS, Y.; KLEINBING, H.; SCRIBELK, L.; VOERKEL, P. - Platz zum Denken!?

Didática de Línguas Estrangeiras Modernas - Alemão, das im Semester 2017-2 im Lehramtsstudiengang Deutsch an der UFRJ angeboten wurde. Das Seminar wurde von etwa zehn Studierenden besucht und im Umfang von zwei Semesterwochenstunden von zwei Lehrkräften im Team-Teaching gestaltet.

Ziel der beiden Dozenten war es, gemeinsam mit den Studierenden verschiedene Zugänge zu gängigen Theorien der Deutschdidaktik zu erarbeiten. Der Ansatz, der dabei auf das größte Interesse stieß, war die Aktionsforschung, weil die Studierenden hier die Forderung wiedererkennen konnten, die im Unterricht vorhandenen Spielräume auszufüllen, das Klassenzimmer nach ihren Vorstellungen zu gestalten und bewusst für eine Verbindung zwischen Theorie und Praxis zu nutzen (vgl. SCHART; LEGUTKE 2012: 63, 149). Dabei sollte die Theorie Eingang ins Unterrichtsgeschehen finden, und der partizipative Ansatz auch bei der Evaluierung am Ende des Semesters zum Tragen kommen. Dafür musste ein passendes Format gefunden werden.

Ein Dozent des Fachs hatte seit mehreren Jahren als Tutor, Prüfer und Coach im von der Universität Jena und dem Goethe-Institut angebotenen Weiterbildungsstudium Deutsch unterrichten gearbeitet und ist deswegen bestens mit den Materialien der Reihe Deutsch lehren lernen (DLL) vertraut ${ }^{9}$. Im Seminar wurde die Idee der in DLL verwendeten Praxiserkundungsprojekte (im Weiteren: PEP) vorgestellt und gemeinsam mit den Studierenden beschlossen, dieses Instrument auch im Seminarverlauf auszuprobieren und für die abschließende Evaluierung zu nutzen.

Das PEP-Format ist insofern hilfreich, als es auf anschauliche, greifbare und realistische Weise der Forderung der Aktionsforschung nach einer Strukturierung der Beobachtung und Reflexion nachkommt (vgl. WALLACE 2010: 14f.; FELDMEIER 2014: 261; MOHR; SCHART 2016: 295). Im Falle von DLL folgt das PEP fünf nachvollziehbaren Schritten:

1) Formulierung der Fragestellung für die Praxiserkundung

2) Darstellung des Impulses für die Fragestellung und Kontextualisierung der Frage im Fachgebiet bzw. im Unterricht

3) Beschreibung und Begründung des Vorgehens, das für die Praxiserkundung gewählt wurde

\footnotetext{
${ }^{9}$ Weitere Informationen zum Weiterbildungsstudium finden sich unter dem Link: http://www.dafdaz.unijena.de/deutsch_unterrichten.html (Zugriff: 11.08.2018)

Pandaemonium, São Paulo, v. 22, n. 36, jan.-abr. 2019, p. 178-206
} 
SANTOS, Y.; KLEINBING, H.; SCRIBELK, L.; VOERKEL, P. - Platz zum Denken!?

4) Aufarbeitung, Analyse und Präsentation Ergebnisse der Beobachtung

5) Reflexion der Praxiserkundung und der Folgen für das unterrichtliche Handeln

Im Laufe der Lehrveranstaltungen wurden diese fünf Punkte nach und nach besprochen und die Studierenden dabei begleitet, die notwendigen Schritte sowohl in der theoretischen Vorbereitung als auch in der praktischen Umsetzung zu gehen. Wichtig war dabei aus Dozentensicht eine flache Progression, da die Studierenden zunächst mit dem für sie ungewohnten Format vertraut werden mussten (vgl. MOHR; SCHART 2016: 301f.). Nach anfänglichem Zögern wurde die Idee, das eigene Handeln mithilfe des PEPs systematisch zu beobachten und zu reflektieren, aber mit zunehmender Begeisterung angenommen und genutzt. Besonders attraktiv erschien den Studierenden, dass die Praxiserkundung auch im Team umgesetzt werden konnte und damit Ansätze der kollegialen Unterrichtsbeobachtung und der kooperativen Lernens zum Tragen kamen (vgl. SCHART; LeGUTKE 2012: 164, MOHR; SCHART 2016: 296, BONNET; DECKE-CORNILL; HERICKS 2017: 173). Aus der Situation der Zusammenarbeit in einer Dreiergruppe heraus entstand letztendlich auch die Idee für den vorliegenden Artikel $^{10}$.

Der Aspekt aus dem DaF-Unterricht, den wir für dieses Projekt ausgewählt haben, war die Arbeit mit Wortschatz. Einerseits ist dieses Thema ein „Dauerbrenner“ in der Unterrichtsrealität, andererseits lassen sich in der Wissenschaft zahlreiche Belege für die hohe Relevanz der Wortschatzarbeit finden (vgl. z. B. NeVELING 2017: 381). Zudem zeigen neuere Forschungsergebnisse, so bspw. zur neuronalen Vernetzung, wie stark die Wörter aufeinander aufbauen. Dabei gilt der Satz von Kühn (2013: 157): ,Je mehr Wörter ein Schüler kennt, desto einfacher ist es, neue dazuzulernen nach dem Motto: Ein Wort gibt das andere.“ Dieses Zitat belegt die Wichtigkeit von Wortschatzarbeit, besonders in einem kommunikativen Kontext. Deshalb fanden wir es wichtig, mit diesem Thema zu arbeiten.

Nachdem wir diese Entscheidung getroffen haben, überlegten wir: Wie können wir als Lehrer den Wortschatz besser erklären? Welches Verfahren ist effektiv, um die Lerner dabei zu unterstützen, den Wortschatz nicht zu vergessen und ihn auch aktiv benutzen zu können? Dazu fanden wir verschiedene Techniken und Theorien: die

\footnotetext{
${ }^{10}$ Die nun folgenden Abschnitte spiegeln einige der Aktivitäten des angebotenen Seminars und geben die Überlegungen aus Sicht der drei beteiligten Studierenden wieder. Aus diesem Grund erfolgt an dieser Stelle auch der Wechsel in die erste Person Plural.
}

Pandaemonium, São Paulo, v. 22, n. 36, jan.-abr. 2019, p. 178-206 
SANTOS, Y.; KLEINBING, H.; SCRIBELK, L.; VOERKEL, P. - Platz zum Denken!?

Übersetzungsmethode, Pantomime, Gestik, Mimik, usw. Ziel war es nun, die verschiedenen Möglichkeiten auszuprobieren und dabei der Frage nachzugehen: Welche von diesen Techniken ist die beste Unterstützung für die Lerner? Wie effektiv können Mimik, Gesten, usw. sein?

Bei der Auswahl der zu verwendenden Techniken hielten wir uns wiederum an die Fachliteratur. So heißt es dort z. B. im Zusammenhang mit der Präsentation von Wortschatz:

Die Wahl des jeweiligen Verfahrens ist abhängig von der Art der Wörter (z.B. abstrakte Wörter, Verben, Bezeichnung von Gegenständen), den Lernern (z. B. Sprachniveau, Lernpräferenzen, Herkunftssprachen, weitere Sprachkenntnisse), dem Lehrenden (zeichnerische Fähigkeiten, darstellerische Fähigkeiten, verbale Fähigkeiten, Sprachkenntnisse), den vorhandenen Materialien und Medien (z. B. Realien, Bilder, Tondokumente). (STORK 2010: 105)

Dieses Zitat belegt, wie stark der Wortschatzerwerb von verschiedenen Faktoren beeinflusst werden kann. Daraus entwickelten wir zwei Fragen, eine allgemeinere und eine sehr konkrete, denen wir in der Praxis nachgehen wollten: Wie können wir mit dem Thema „Wortschatz“ arbeiten, um diesen möglichst effektiv und gemäß den Lernerinteressen zu vermitteln? Und: Mit welchen Wörtern sollten wir uns beschäftigen: mit abstrakten oder konkreten Wörtern?

Um ein möglichst repräsentatives Ergebnis zu erreichen, benutzten wir in unserem Projekt abstrakte Wörter, weil wir der Meinung sind, dass es schwieriger ist, mit dieser Art von Vokabeln zu arbeiten. Zur Anwendung kamen zwei verschiedene Erklärungsverfahren: das teilweise nichtsprachliche Erklärungsverfahren (durch die gegenständliche Veranschaulichung, bildliche Veranschaulichung, Gestik / Mimik / Pantomime, Klangbilder) und das zweisprachige Verfahren (durch die Übersetzung, Wortähnlichkeiten zwischen Mutter- und Fremdsprache, Wortähnlichkeiten zwischen erster und zweiter Fremdsprache, Internationalismen).

Was die Umsetzung des Projekts angeht, so ist klarzustellen, dass die Planung und Ausarbeitung gemeinsam erfolgte, die Umsetzung jedoch individuell. Für die Umsetzung des Projekts wurde entschieden, die Verfahren in Deutschkursen auf drei verschiedenen Niveaustufen auszuprobieren. Deshalb wurde der Projektversuch zur Wortschatzarbeit im November 2017 mit Deutsch 1 (Niveau A1.1), Deutsch 2 (Niveau A1.2) und Deutsch 3 (Niveau A2.1) durchgeführt. Die Niveaus folgen dem Gemeinsamen europäischen Referenzrahmen für Sprachen (GeR), aber das CLAC hat seine eigene Methodologie, 
SANTOS, Y.; KLEINBING, H.; SCRIBELK, L.; VOERKEL, P. - Platz zum Denken!?

Bewertungskriterien und passenden Inhalt. Laut dem CLAC-Katalog 2017-2018 liegt der Schwerpunkt im Kurs Deutsch 1 auf dem , [...] Verständnis und Verwendung von alltäglichen Ausdrücken [...]. Es wird erwartet, dass der Lernende einfach kommunizieren kann“. ${ }^{11}$ In Deutsch 2 geht es um eine Vertiefung und Erweiterung des Wortschatzes, den die Lerner beherrschen sollen (u. a. zu den Themen Beruf, Einkaufen, Lebensmittel, Körperteile und Kleidung). Da die Lernenden in Deutsch 3 schon viel mehr Kontakt mit der Zielsprache hatten, sind sie am ehesten damit vertraut, häufig vorkommende, einfache Ausdrücke zu verstehen.

Im Laufe der Projektplanung stellten wir fest, dass unsere Klassen eine ähnliche Zusammensetzung von Kursteilnehmern aufweisen. Die meisten Lernenden sind Studierende aus der UFRJ und nehmen das Fremdsprachenangebot innerhalb der Institution wahr. Gleichzeitig gibt es auch einige externe Lerner, die mit einer ähnlichen Motivation kommen: Der Preis des Kurses ist nicht hoch, sie interessieren sich für mögliche Austauschmaßnahmen und Stipendien nach Deutschland, und sie vertreten die unterschiedlichsten Fachrichtungen. Vom Alter her überwiegt die Gruppe der 20- bis 30jährigen, es handelt sich also um (junge) Erwachsene.

Ein Unterschied war das Niveau der Lerner in den verschiedenen Kursen (Niveaus A1.1, A1.2 und A2.1), auf das jeweils spezifisch eingegangen werden musste. Das heißt, die Art der angebotenen Aktivitäten war gleich, aber jede Lehrkraft hat ihre eigene Adaptation vorgenommen, damit das Angebot zum jeweiligen Niveau passt. Dies gilt auch für den zu vermittelnden Wortschatz, der sich an Wortlisten aus dem Lehrwerk in Anlehnung an den GeR orientierte. Am Ende jeder Aktivität füllten die Teilnehmer eines jeden Kurses einen Fragebogen aus, sodass die Kursleiter die Schwierigkeiten der angebotenen Unterrichtsaktivitäten analysieren konnten und daraus deutlich wurde, welche Art der Übung am positivsten bewertet wurde. Trotz der Niveauunterschiede wurde derselbe Fragebogen eingesetzt (siehe Anhang 1). Dieser wurde gemeinsam erstellt und (weitgehend auf Portugiesisch, der L1 der Lerner) ausformuliert, um vergleichbare Ergebnisse zu bekommen.

In den nächsten Teilkapiteln werden sowohl die Aktivitäten als auch die erzielten Ergebnisse vorgestellt, die uns letztlich zu unseren Schlussfolgerungen veranlassen.

\footnotetext{
${ }^{11}$ Übersetzung der Verfasser. Im Original steht: "O foco desse nível é a compreensão e o uso de expressões cotidianas [...] nível, espera-se que o aprendiz consiga se comunicar de modo simples".

Pandaemonium, São Paulo, v. 22, n. 36, jan.-abr. 2019, p. 178-206
} 


\subsection{Vorgehen in Gruppe 1}

Die Unterrichtssequenz fand mit dem Niveau A1.1 und 17 Kursteilnehmern statt. Die Arbeit mit dem Wortschatz umfasste hier insgesamt 10 verschiedene Wörter zum Thema Feiern, die in zwei Gruppen aufgeteilt wurden. Eine Gruppe bestand aus 5 Wörtern (Weihnachten, Verlobung, Ostern, Silvester und Hochzeit), die den Lernern durch Mimik, Gesten und Zeichnungen erklärt wurden. Bei der anderen Gruppe von Wörtern (Geburtstag, Neujahr, Pfingsten, Erntedankfest und Oktoberfest) wurde mit einer direkten Übersetzung ins Portugiesische gearbeitet.

Die Unterrichtsstunde begann mit einer kurzen Einführung zu Lektion 12 des Buchs „Menschen A1.1“, in der es um das Thema Feiern geht. Danach wurden die Regeln für die Unterrichtssequenz erklärt: die Kursteilnehmer durften die Übersetzung nicht laut sagen und Wörterbücher waren verboten. Die Wörter wurden nur durch Mimik und Gesten erklärt, aber diese konnten mehrmals wiederholt werden. Dann wurden die ersten fünf Wörter an die Tafel geschrieben. Jedes Wort wurde durch die Kursleiterin mithilfe von Mimik und Gesten semantisiert, und die Kursteilnehmer sollten ihre Bedeutung in ihr Heft schreiben. Nach diesem ersten Teil der Aktivität ging der Unterricht wie sonst üblich weiter.

Der zweite Teil der Aktivität wurde nach der Pause durchgeführt, eine Vokabelarbeit mit der direkten Übersetzung ins Portugiesisch. Dazu wurden die anderen fünf Wörter an die Tafel geschrieben und die Kursleiterin stellte den Teilnehmern Fragen: „Was bedeutet Pfingsten auf Portugiesisch?“ oder „Wer von euch hat im Dezember Geburtstag? Welche Feste haben wir im Dezember?“. Wenn ein Teilnehmer das Wort nicht kannte, wurde es ins Portugiesische übersetzt. Nach diesem Teil der Aktivität ging der Unterricht normal weiter.

Kurz vor dem Ende der Unterrichtsstunde wurden den Kursteilnehmern zwei Aufgaben mit Bezug zur Wortschatzarbeit vorgelegt. Die erste Aufgabe war ein Lückentext zu den ersten fünf Wörtern, und in der zweiten Aufgabe sollten die Lerner verschiedene Sätze mit den Modalverben mögen und können bilden, in denen sie die Wörter aus dem zweiten Teil der Aktivität verwendeten. 


\section{1) Ergänzen Sie bitte den Lückentext:}

das Weihnachten - die Verlobung - das Ostern - das Silvester - die Hochzeit

a) Jedes Jahr an besucht der Osterhase die Kinder.

b) ist das Versprechen, eine Person zu heiraten.

c) Der letzte Tag des Jahres bezeichnet am 31. Dezember.

d) ist das Fest der Geburt von Jesus Christus. Festtag ist der 25. Dezember.

Abbildung 1: Lückentext zum Ergänzen (Gruppe 1)

\subsection{Vorgehen in Gruppe 2}

Die Unterrichtssequenz fand mit dem Niveau A1.2 und 11 Kursteilnehmern statt. Auch hier stellte sich die Frage nach einem adäquaten Vermittlungskontext. Passenderweise hatte die Lektion 24 im Buch „Menschen A1.2“ - ebenso wie die behandelte Lektion in Gruppe 1 - das Thema Feiern zum Thema (Titel: „Ich würde am liebsten jeden Tag feiern“).

Die Unterrichtssequenz wurde für zwei verschiedene Phasen geplant. Im ersten Teil sollten die Vokabeln durch Mimik und Gestik erklärt werden. Dazu wurden die folgenden Wörter ausgewählt: Weihnachten, Verlobung, Ostern, Silvester und Hochzeit. In dem anderen Teil sollte das Vokabular nur übersetzt werden und die ausgewählten Wörter waren: Einweihungsparty, Erntedankfest, Geburtstag, Pfingsten und Neujahr ${ }^{12}$.

Am Anfang des Unterrichts wurde über das Oktoberfest gesprochen, weil die Lerner schon von diesem Fest gehört hatten - es ist in Brasilien recht bekannt und zudem Inhalt in Kurs 1. Das war eine gute Einführung, und nach dieser Phase wurden die neuen

\footnotetext{
${ }^{12}$ Da die Thematik der bearbeiteten Lehrbucheinheit sich ähnelte, haben wir uns dazu entschieden, trotz der Niveauunterschiede in Gruppe 1 und Gruppe 2, mit ähnlichen Vokabeln zu arbeiten - lediglich die Wörter Einweihungsparty und Oktoberfest differieren.
}

Pandaemonium, São Paulo, v. 22, n. 36, jan.-abr. 2019, p. 178-206 
SANTOS, Y.; KLEINBING, H.; SCRIBELK, L.; VOERKEL, P. - Platz zum Denken!?

Wörter zum Thema Feiern an die Tafel geschrieben und semantisiert. Dazu wurde ein Mimik-Spiel gemacht. Vor der Aktivität mit Mimik und Gesten wurden die Regeln erklärt: Normalerweise haben die Lerner die Gewohnheit, die Übersetzung der Wörter laut zu sagen, aber diesmal sollten sie die Bedeutung für sich behalten, egal ob schriftlich oder nur im Kopf.

Der zweite Teil der Unterrichtssequenz wurde nach der Pause durchgeführt und die Lerner hatten das Buch schon geöffnet. Damit konnten sie einige andere Wörter im Bildlexikon des Buches sehen. Daneben wurde der Wortschatz an die Tafel geschrieben und die Wörter wurden direkt übersetzt. Nach der Erklärung des Wortschatzes bekamen die Lerner zwei Aufgaben vorgelegt. Die erste war ein Lückentext ${ }^{13}$, den sie mit den neuen Wörtern ergänzen sollen. Dazu durften sie ihre Notizen individuell benutzen.

Die zweite Aktivität fand am Ende des Unterrichts statt. Dabei sollten die Lerner die anderen Wörter benutzen, um irreale Sätze mit dem Verb würden zu bilden. Um die Aufgabe deutlicher zu machen, gab es Beispiele, wie: „Zu Neujahr würde ich viel trinken“, oder: „Ich würde eine Einweihungsparty mit meinen Freunden machen“.

\subsection{Vorgehen in Gruppe 3}

Die Unterrichtssequenz wurde mit dem Niveau A2.1 und 21 Kursteilnehmern durchgeführt. Hier gab es acht abstrakte Wörter, die in zwei Phasen vermittelt wurden. Die erste Phase bestand aus der Einführung von vier Wörtern (Irrtum, Abscheu, Einbildung und Schadenfreude), die durch ein teilweise nichtsprachliches Erklärungsverfahren erklärt wurden. Dabei wurden die Wörter durch Mimik, Synonyme, Beispiele usw. eingeführt, wobei die Erklärungen konsequent einsprachig auf Deutsch erfolgten. Die Kursteilnehmer hatten einige Schwierigkeiten, die Bedeutungen zu verstehen, da diese nicht unmittelbar erkennbar war. Die Schwierigkeiten wurden bei der Übung, die sie im Anschluss machen sollten, deutlich: sie sollten die Wörter mit ihren jeweiligen Bedeutungen anpassen. Die zweite Phase bestand aus den anderen vier Wörtern (Abenteuer, Mitleid, Eitelkeit und Erschöpfung), bei deren Semantisierung die Einsprachigkeit aufgehoben wurde. In dieser Phase wurden die Wörter direkt ins Portugiesische übersetzt und dies gab den Kursteilnehmern viel mehr Selbstvertrauen, um

\footnotetext{
${ }^{13}$ Es handelte sich dabei um denselben Lückentext wie bei Gruppe 1 (vgl. Abbildung 1).

Pandaemonium, São Paulo, v. 22, n. 36, jan.-abr. 2019, p. 178-206
} 
SANTOS, Y.; KLEINBING, H.; SCRIBELK, L.; VOERKEL, P. - Platz zum Denken!?

die Übung zu lösen. Dies wurde bei der Korrektur der Aktivität, die in der folgenden Abbildung gezeigt wird, deutlich.

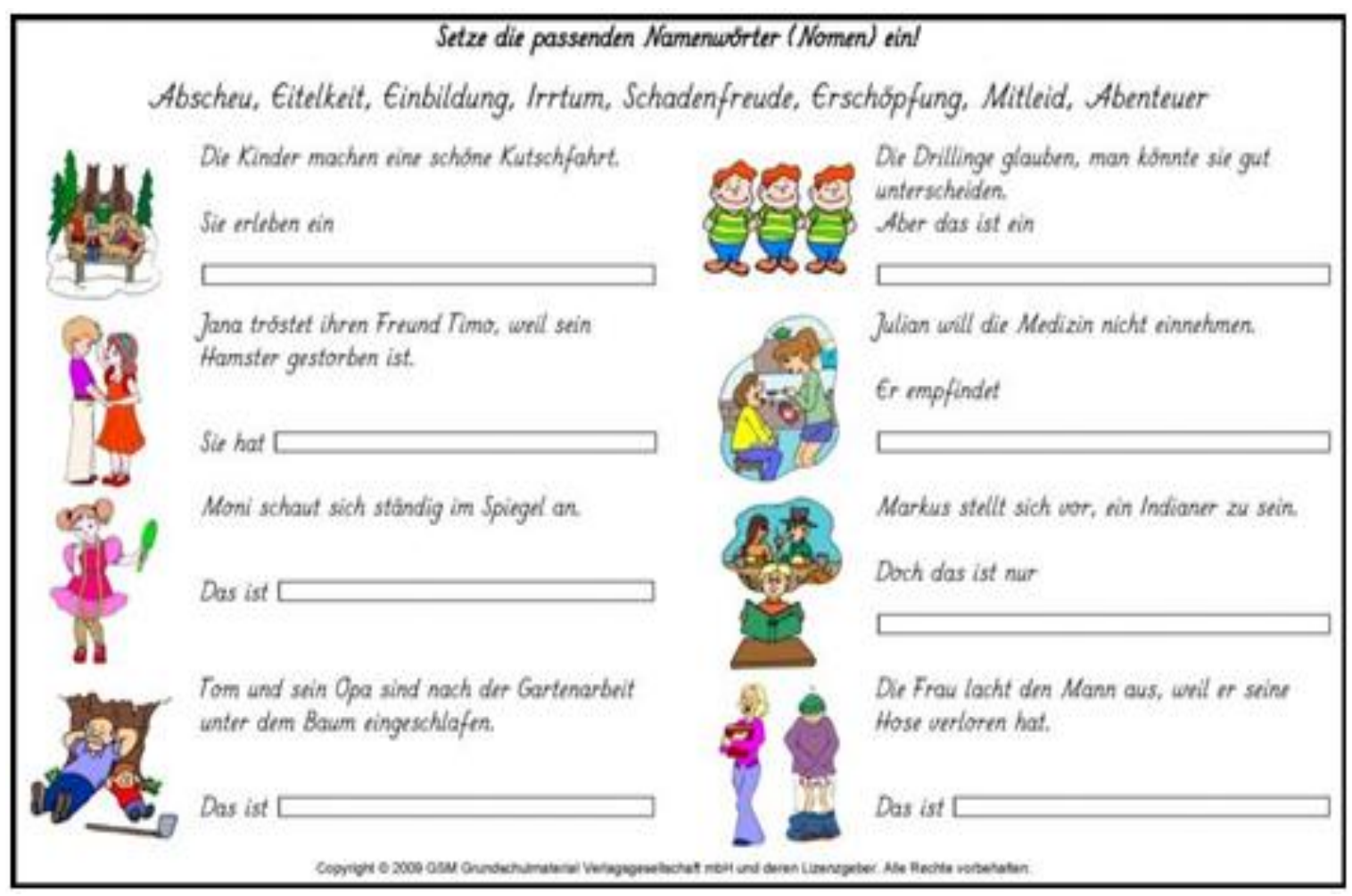

Abbildung 2: Lückentext zum Ergänzen (Gruppe 3)

Jede Phase der Unterrichtssequenz bestand aus zwei Teilen: Im ersten Teil sollte ich als Lehrerin die abstrakten Wörter erklären. Dann sollten die Kursteilnehmer die Übung machen. Nach diesem ersten Teil lief der Unterricht wie gewohnt weiter. Im zweiten Teil sollten die Kursteilnehmer Sätze schreiben, in denen sie bekannte grammatische Formen verwenden sollten (Sätze mit wenn), um die abstrakten Wörter zu benutzen. Die Sätze sollten in Verbindung mit ihrem Umfeld stehen. Zum Beispiel: „Wenn ich Salat esse, fühle ich Abscheu.“ Die Absicht war, dass sie die Sätze frei schreiben konnten, indem sie ihnen bereits bekannte grammatische Formen anwendeten, wodurch ihnen der Gebrauch der neu gelernten abstrakten Wörter erleichtert würde. Dies bietet auch mehr Freiheit für Fehler, im Gegensatz zu der ersten Aktivität, die viel geschlossener gehalten war. 


\section{Ergebnisse der Untersuchung}

Dass nicht jeder Lerner gleich ist, wird bereits im Unterricht schnell deutlich. Deshalb ist die Aufmerksamkeit der Lehrer den Schülern gegenüber auch so wichtig. Der Anspruch, die Perspektive der Lerner in die Untersuchung mit einzubeziehen, motivierte uns zur Erstellung des Fragebogens, mithilfe dessen wir uns - zunächst aus subjektiver Sicht weitere Ergebnisse zum Wortschatzerwerb erhofften. Die Vorbereitung von gleichen Fragebögen für die drei Versuchsgruppen ermöglichte einen organisierten Überblick, sowohl in Hinblick auf die Sprachschüler als auch auf die Lehrer. Dazu ist es wichtig zu sagen, dass jeder Lehrende seine eigene Unterrichtsplanung und -interaktion umgesetzt hat. Die Unterrichtsinhalte waren - aufgrund der unterschiedlichen Niveaustufen - nicht identisch, so dass bereits dadurch unterschiedliche Ergebnisse zu erwarten waren. Gleichzeitig war in allen drei Gruppen darauf geachtet worden, zwei unterschiedliche Vermittlungstechniken für den Wortschatz zu benutzen, so dass beobachtet werden konnte, welches Vorgehen jeweils besser zur Gruppe passte und wie der Prozess des Wortschatzlernens als einfacher wahrgenommen würde.

Für den Prozess der Beobachtung war die Beschäftigung mit der Aktionsforschung essenziell. Wir nutzten dabei die Möglichkeit, den Unterricht als ein Versuchslabor zu betrachten, als einen Raum, um Erfahrungen zu sammeln (vgl. SCHART; LEGUTKE 2012: 71). Die Erfahrungen wurden dabei systematisiert und folgten einer bestimmten Struktur: Aus dem Unterrichtsgeschehen heraus wurde eine Schwierigkeit identifiziert (das Wörterlernen), dazu eine konkrete Fragestellung erarbeitet und eine Unterrichtssequenz in drei Vergleichsgruppen geplant und durchgeführt. Wichtig in allen Phasen war der intensive Austausch untereinander sowie die gemeinsame Reflexion (vgl. MOHR; SCHART 2016: 295f.). Die Ergebnisse lassen sich - neben den eigenen Beobachtungen - vor allem mittels des Fragebogens ablesen, den die Kursteilnehmer im Anschluss an die Unterrichtssequenz ausfüllten.

Der Fragebogen selbst umfasste drei Fragen. In der ersten Frage ging es um die Einschätzung der ersten Lernaktivität der Unterrichtssequenz (Erklärung auf Deutsch durch Synonyme, Beispiele, Mimik, Gesten usw.), die zweite Frage bezog sich auf die zweite Aktivität (Semantisierung mittels direkter Übersetzung) - beides jeweils in Hinblick auf den Verstehensprozess und die damit verbundenen Schwierigkeiten. Die dritte Frage war eine Einschätzung der gesamten Unterrichtssequenz: die Kursteilnehmer Pandaemonium, São Paulo, v. 22, n. 36, jan.-abr. 2019, p. 178-206 
SANTOS, Y.; KLEINBING, H.; SCRIBELK, L.; VOERKEL, P. - Platz zum Denken!?

sollten entscheiden, welche Aktivität aus ihrer Sicht am produktivsten war und welche Art der Vermittlung sie bevorzugen. Die Ergebnisse aus den Fragebögen werden im Folgenden dargestellt.

Im Kurs Deutsch 1 (Niveau A1.1) wählten in Bezug auf die erste Aktivität fünf Kursteilnehmer sehr einfach, neun einfach und drei mittel. In Bezug auf die zweite Aktivität wurde von fünf Kursteilnehmern sehr einfach ausgewählt, von vier einfach, von sechs mittel und von zweien schwierig.

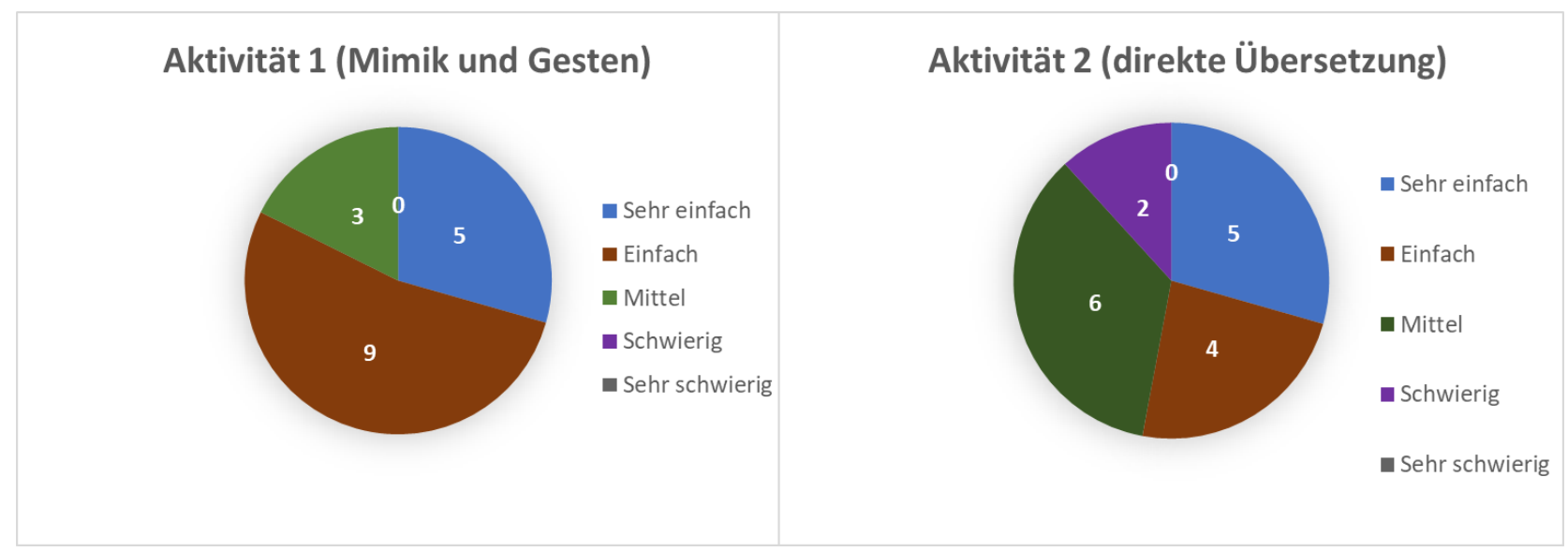

Grafik 1: Ergebnisse des Fragebogens (Gruppe 1)

Im Kurs Deutsch 2 (Niveau A1.2) antworteten in Bezug auf die erste Aktivität vier Kursteilnehmer sehr einfach, vier einfach und drei mittel. In Hinblick auf die zweite Aktivität wurde von vier Kursteilnehmern sehr einfach ausgewählt, von sechs einfach und von einem mittel.

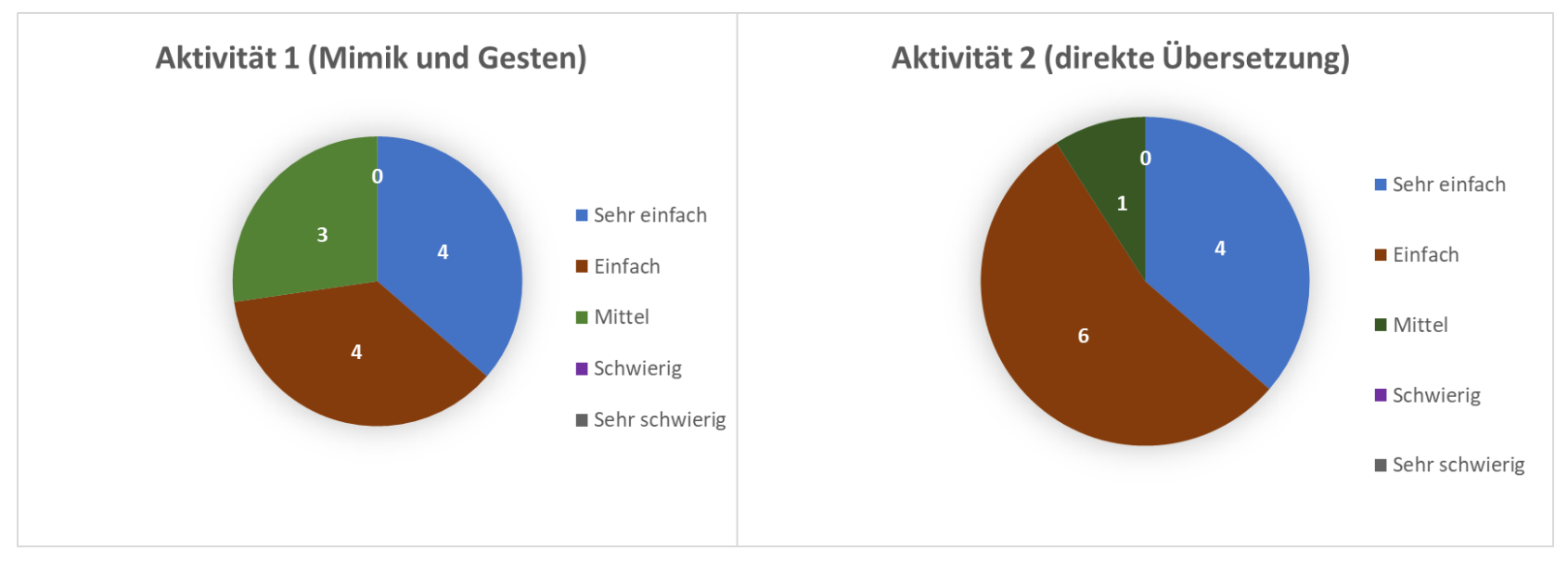

Grafik 2: Ergebnisse des Fragebogens (Gruppe 2) 
SANTOS, Y.; KLEINBING, H.; SCRIBELK, L.; VOERKEL, P. - Platz zum Denken!?

Im Kurs Deutsch 3 (Niveau A2.1) antworteten in Bezug auf die erste Aktivität drei Kursteilnehmer sehr einfach, elf einfach und sieben mittel. In Bezug auf die zweite Aktivität wurde von zwölf Kursteilnehmern sehr einfach ausgewählt, von fünf einfach und von vier Lernern mittel.

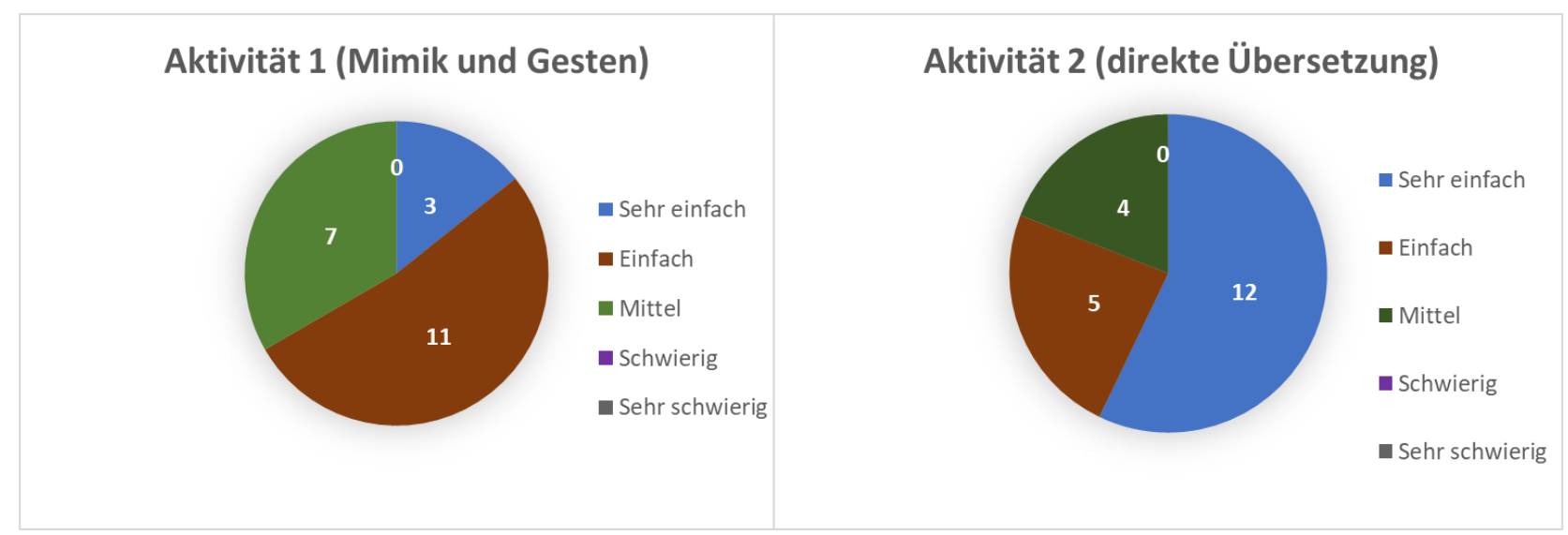

Grafik 3: Ergebnisse des Fragebogens (Gruppe 3)

Im Folgenden werden die Ergebnisse der dritten Frage vorgestellt. Dabei wurde jeweils unterschieden, welche Aktivität den Lernern am besten gefallen hat, und welche von ihnen als produktiver (d. h. als sinnvoller für den Lernprozess) wahrgenommen wurde.

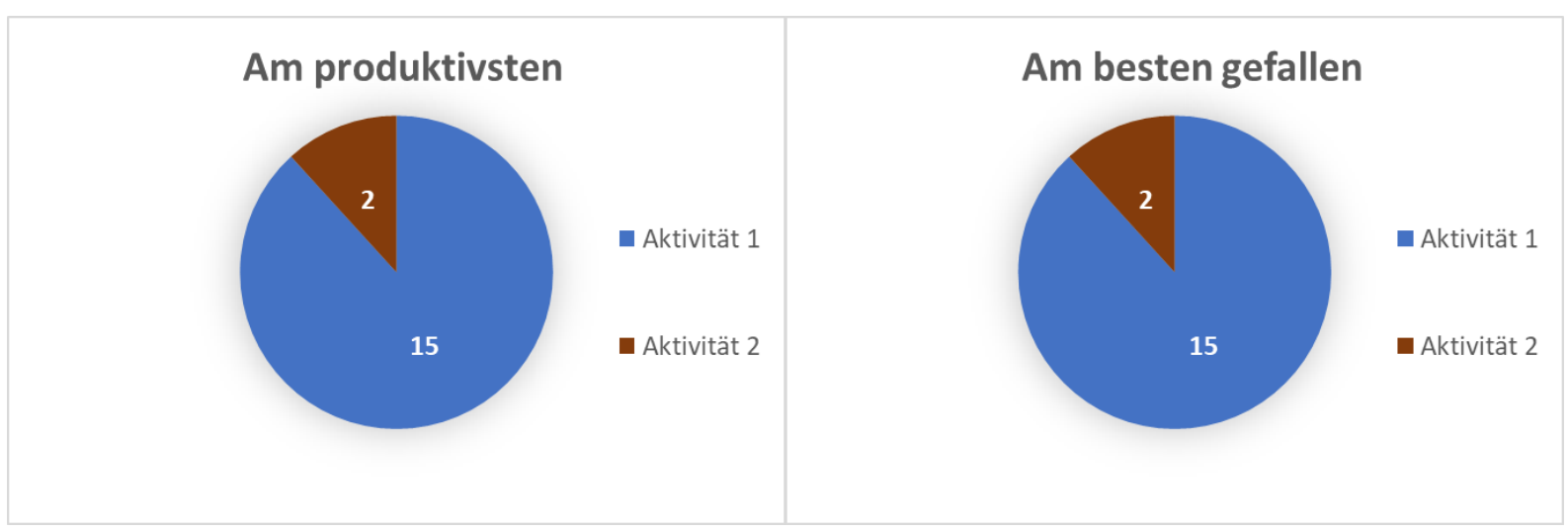

Grafik 4: Ergebnisse des Fragebogens, Frage 3 (Gruppe 1) 
SANTOS, Y.; KLEINBING, H.; SCRIBELK, L.; VOERKEL, P. - Platz zum Denken!?

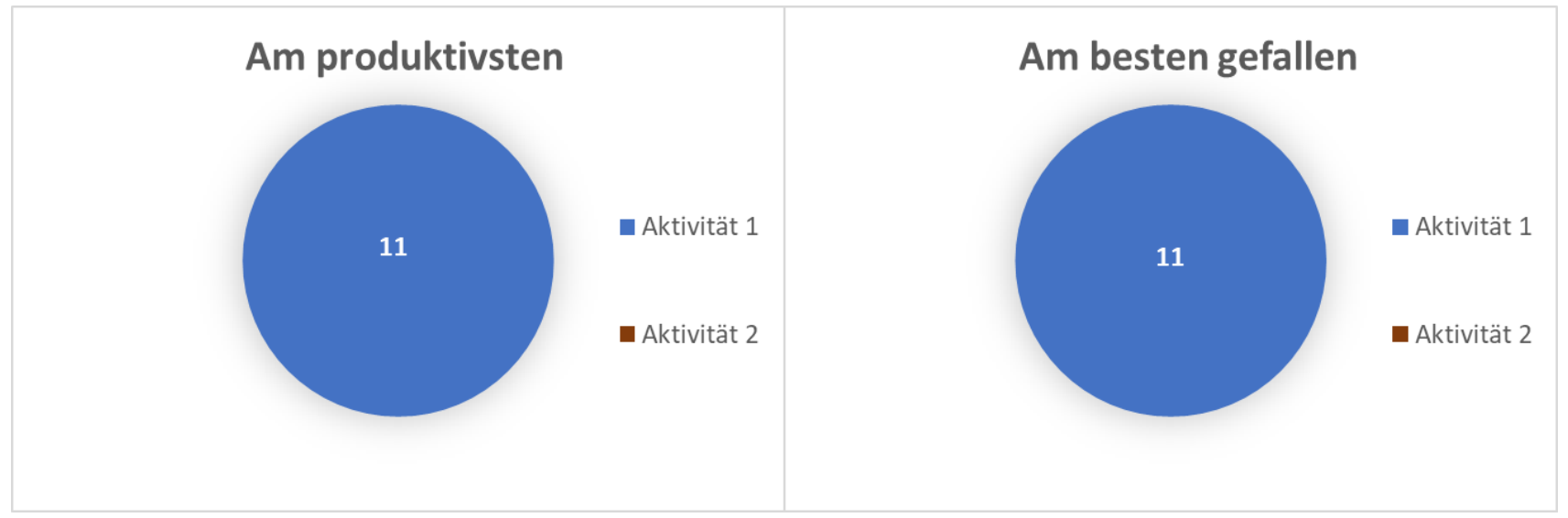

Grafik 5: Ergebnisse des Fragebogens, Frage 3 (Gruppe 2)

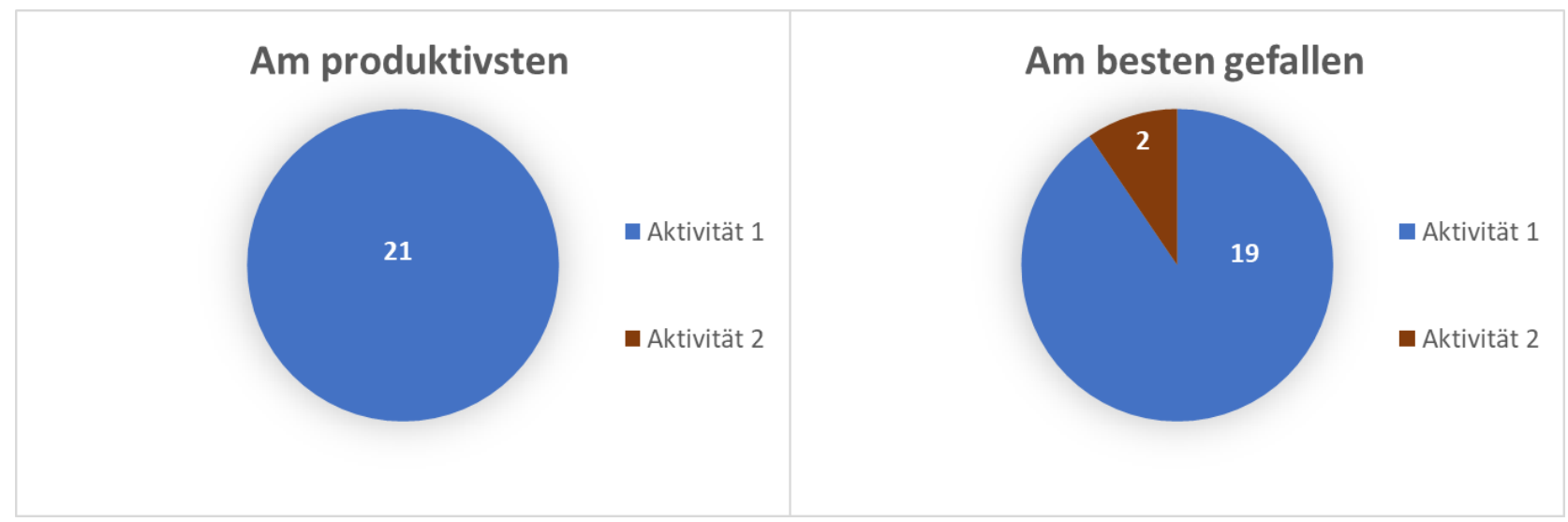

Grafik 6: Ergebnisse des Fragebogens, Frage 3 (Gruppe 3)

Beim der Analyse der Grafiken konnten wir feststellen, dass es unter den 49 befragten Kursteilnehmern (17 aus Deutsch 1, elf aus Deutsch 2 und 21 aus Deutsch 3) eine klare Präferenz für die erste Aktivität gab, bei der es um die Vermittlung von Wörtern über Mimik und Gestik ging. Dieses Ergebnis war aus unserer Sicht eine positive Überraschung, weil wir damit gerechnet hatten, dass viele der Befragten die zweite, ihnen klar vertrautere zweite Aktivität auswählen würden. Stattdessen wurde deutlich, dass die Kursteilnehmer keine wesentlichen Schwierigkeiten mit der neuen Art der Vermittlung hatten und diese mehrheitlich auch als produktiver erachteten. Damit konnte gezeigt werden, dass der Gebrauch von nonverbalen Lerntechniken wie Mimik und Gestik nach Auffassung der Lerner besser und produktiver sei als die Nutzung traditioneller Techniken wie die direkte Übersetzung. Zwischen den verschiedenen Kursniveaus waren bei den Einschätzungen der Lerner keine wesentlichen Unterschiede erkennbar. 
SANTOS, Y.; KLEINBING, H.; SCRIBELK, L.; VOERKEL, P. - Platz zum Denken!?

An dieser Stelle soll noch einmal betont werden, dass es sich bei dem von uns durchgeführten Praxiserkundungsprojekt nicht um eine empirische Forschung im klassischen Sinne handelt, bei der die Ergebnisse allgemeine und valide Aussagen über die Effizienz bestimmter Vermittlungsstrategien und -techniken generieren sollen ${ }^{14}$. Dies wäre im von uns genutzten Setting auch kaum möglich, ist der Lernprozess doch durch seinen multifaktoriellen Charakter gekennzeichnet und verschiedensten Einflüssen unterworfen. Vielmehr geht es um das Ausprobieren bestimmter Theorien in der Praxis und das Einüben eines strukturierten Vorgehens bei der Reflexion von Unterrichtsprozessen. In diesem Sinne ist das Ziel der Praxiserkundung durchaus erreicht worden: Davor waren wir der Meinung gewesen, dass die direkte Übersetzung von unbekannten Wörtern ins Portugiesische den Kursteilnehmern besser helfen würde. Die Lerner erklären zwar, dass die Sinnübertragung mittels direkter Übersetzung schnell und einfach verläuft, das Vergessen der Wörter jedoch auf gleiche Weise passiert: schnell und einfach.

Mit dieser Praxiserkundung und ihren Ergebnissen fanden wir bestätigt, wie wichtig es für uns als Lehrer ist, die Meinung der Kursteilnehmer und ihre Lernpräferenzen kennenzulernen, weil es sich um ganz relevante Faktoren im Unterrichtsverlauf handelt. Es war zudem eine positive Erfahrung für uns zu sehen, dass auch diejenigen Kursteilnehmer, die fast nichts über Lernstrategien wussten, ein Gefühl dafür hatten, welche Methode ihrem Verständnis besser hilft und damit sinnvoller für sie ist.

\section{Abschließende Überlegungen}

Die Erfahrungen, die wir an der Bundesuniversität von Rio de Janeiro gesammelt haben, zeigen uns, dass das Curriculum des Lehramtsstudiengangs die Studierenden nur unzureichend auf ihre Tätigkeit als Lehrperson vorbereitet. Ein erkennbarer Mangel besteht im Fehlen von Praktikumsmöglichkeiten während des Studiums, wodurch erschwert wird, Lehrerfahrungen $\mathrm{zu}$ sammeln und sich erste Unterrichtsroutinen anzueignen. Dieser Mangel hat zum Teil negative Auswirkungen auf die Motivation der

\footnotetext{
${ }^{14}$ Diese Einstellung wird bereits über die Wortwahl deutlich - Erkundung statt Erforschung - und ist in der Forschungsliteratur verschiedentlich erläutert worden (vgl. u. a. SCHART; LEGUTKE 2012: 186, MOHR; SCHART 2016: 292f.).
}

Pandaemonium, São Paulo, v. 22, n. 36, jan.-abr. 2019, p. 178-206 
SANTOS, Y.; KLEINBING, H.; SCRIBELK, L.; VOERKEL, P. - Platz zum Denken!?

Studierenden - auf der anderen Seite kann er auch dazu führen, dass die Betroffenen selbst aktiv werden. Eine Lösung ist es, andere Unterrichtsorte zu suchen, um Erfahrung beim Lehren zu sammeln und damit eine Verbindung zwischen Theorie und Praxis herzustellen. Im Falle der UFRJ besteht mit dem CLAC auch für Studierende schon die Möglichkeit, Sprachlehrer zu sein. Die spezifische Struktur des CLAC als Projeto de Extensão eröffnet den Lehrkräften dabei Freiheiten, die sie möglicherweise an Sprachschulen o. ä. nicht hätten: insbesondere die Gelegenheit, verschiedene Dinge auszuprobieren und dadurch ihr Lehrprofil auszubauen. Auf diese Weise gewinnt die Praxiskomponente die wichtige Rolle zurück, die sie im Studium haben sollte.

Die Praxiserkundung am CLAC durchzuführen war für uns eine Gelegenheit, die Aktionsforschung an unseren Lehrorten, also in unseren Klassenzimmern, einzusetzen und dadurch bewusst zu reflektieren. Es geht uns darum, in Zukunft nicht nur auf dem Arbeitsmarkt einen Platz $\mathrm{zu}$ finden, sondern die erlernten Theorien kritisch und aufmerksam einzusetzen. Diese Theorien reichen für sich alleine nicht aus, weil sie nicht automatisch für jede Situation passen: Sie müssen getestet werden, damit wir sehen können, inwieweit sie sich für unseren Lehrkontext und in unserer Unterrichtspraxis bewähren.

Wie sinnvoll eine Überprüfung von Theorien und auch eigenen Meinungen über den Unterricht ist, hat uns das PEP klar gezeigt (vgl. MOHR; SCHART 2016: 297). Wir dachten im Vorfeld, alle Kursteilnehmer hätten im Unterricht für Worterklärungen lieber eine direkte Übersetzung als eine Semantisierung mit Mimik und Gesten. Wir waren entsprechend überrascht, als wir die Ergebnisse der Fragebögen analysiert und festgestellt haben, dass es aus Lernersicht produktiver ist, wenn wir Mimik und Gesten benutzen. Ein klares Beispiel für die Diskrepanz zwischen den eigenen Vorstellungen als Lehrkraft und der Position der Lerner und damit ein Plädoyer für ein bewusstes und reflektiertes Vorgehen im Unterricht. Dabei ist es notwendig, keine festen und irreversiblen Meinungen zu bilden, sondern flexibel zu bleiben und die Vorstellungen der Lerner kennenzulernen und sinnvoll in den Unterricht zu integrieren. Das heißt konkret: Während die Erfahrungen dieses PEPs gezeigt haben, dass die große Mehrheit der Lerner den Einsatz von Mimik, Gestik und Zeichnungen befürwortet, kann eine andere Lerngruppe möglicherweise den Einsatz der direkten Übersetzung bevorzugen.

Der gesamte beschriebene Prozess - vom impulsgebenden Seminar über die Praxiserkundung bis hin zur Reflexion - war für uns wertvoll, denn er hat uns dabei 
SANTOS, Y.; KLEINBING, H.; SCRIBELK, L.; VOERKEL, P. - Platz zum Denken!?

unterstützt, einen aktiveren und kritischeren Blick auf den Unterricht (insbesondere die Lehrerrolle) zu entwickeln und einzuüben. Dank der intensiven Vorbereitung, der Unterrichtsgestaltung und den Nachbesprechungen konnten wir unsere Lehrerfahrungen weiter ausbauen. Durch das PEP haben wir zudem erlebt, dass wir schon als angehende Lehrer auch Forscher unseres eigenen Tuns sein können (vgl. MOHR; SCHART 2016: 293).

An dieser Stelle sehen wir auch die Verantwortung der Universität. Diese sollte bei der Ausbildung der Studierenden nicht nur eine spätere Tätigkeit als Forscher im Blick haben, sondern auch ganz dezidiert das Profil als zukünftige Lehrkräfte beachten. Eine intensive Begleitung über Erfahrungen und Reflexion sind dabei unabdingbar (vgl. MOHR; SCHART 2016: 299f., 316).

Eine solche intensive Auseinandersetzung mit einem bestimmten Aspekt des Unterrichtens, wie es bei unserem Projekt der Fall war, ist - sicherlich schon allein aufgrund der personellen Ressourcen - nicht in allen Studienkontexten möglich. Auch muss bedacht werden, dass die Aktionsforschung sicher nicht für alle Studierenden gleich gewinnbringend ist, denn es handelt sich um einen komplexen Prozess, der schnell zu Überforderung führen kann (vgl. BENITT 2015: 74). Trotzdem zeigen unsere Erfahrungen klar in eine Richtung: Platz zum Denken ist für die Lehrerausbildung unentbehrlich, wenn das Ziel ist, die Studierenden auf die Praxis vorzubereiten und ihnen Bewusstsein für ihr Handeln zu vermitteln. Dazu braucht es Raum, sowohl zeitlich als auch physisch, der möglicherweise durch eine (noch) stärkere Berücksichtigung von didaktischen Fragestellungen in den Curricula der Deutschstudiengänge geschaffen werden kann. Ebenso braucht es Gelegenheiten, bei denen die Studierenden sich im Unterrichten ausprobieren können. Und es braucht die Einsicht um die Wichtigkeit der Lehrperson und die Berücksichtigung dieser in Form und Inhalt der Lehrerausbildung (vgl. SCHART; LEGUTKE 2012: 6).

Den eingangs genannten Platz zum Denken versehen wir nun mit einem Ausrufezeichen, denn die eigenen Erfahrungen mit der Praxiserkundung haben uns gezeigt, dass es sich lohnt, sich für diese Art der Verbindung von Theorie und Praxis einzusetzen, da diese tatsächlich umsetzbar und alltagskompatibel ist (vgl. MoHR; SCHART 2016: 294). 


\section{Literaturverzeichnis}

ABENDROTH-TIMMER, Dagmar. Lehrerforschung. In: SURKAMP, Carola (Hg.). Metzler Lexikon Fremdsprachendidaktik. Stuttgart: J. B. Metzler, 2017, p. 196-199.

ALTRICHTER, Herbert; POSCH, Peter. Lehrerinnen und Lehrer erforschen ihren Unterricht. Unterrichtsentwicklung und Unterrichtsevaluation durch Aktionsforschung. Bad Heilbrunn: Klinkhardt, 2007.

AuswäRTIGES AMT. Deutsch als Fremdsprache weltweit. Datenerhebung 2015. Disponível em: <https://www.goethe.de/resources/files/pdf37/Bro_Deutschlernerhebung_final2.pdf> Acesso em 08.06.2018.

BALlWEG, Sandra et al. Wie lernt man die Fremdsprache Deutsch? München: KlettLangenscheidt, 2013.

BENITT, Nora. Becoming a (Better) Language Teacher. Classroom Action Research and Teacher Learning. Tübingen: Narr, 2015.

BoECKMANN, Klaus-Börge. Aktionsforschung. In: BuRWITZ-MElZER, Eva et al. Handbuch Fremdsprachenunterricht. 6. Auflage. Tübingen: A. Francke, 2016, p. 592-597.

BONNET, Andreas; DeCKE-CORNILl, Helene; HERICKS, Uwe. Kooperatives Lernen. In: SuRKAMP, Carola (Hg.). Metzler Lexikon Fremdsprachendidaktik. Stuttgart: J. B. Metzler, 2017, p. 172-174.

DOFF, Sabine. Vermittlungsmethoden: Historischer Überblick. In: BURWITZ-MELZER, Eva et al. Handbuch Fremdsprachenunterricht. 6. Auflage. Tübingen: A. Francke, 2016, p. 320325.

EDMONDSON, Willis; HOUSE, Juliane. Einführung in die Sprachlehrforschung. 4., überarbeitete Auflage. Tübingen: A. Francke, 2011.

FELDMEIER, Alexis. Besondere Forschungsansätze: Aktionsforschung. In: SETTINIERI, Julia et al. (Hg.). Empirische Forschungsmethoden für Deutsch als Fremd- und Zweitsprache. Paderborn: Ferdinand Schöningh, 2014, p. 255-267.

Franco-SANTORO, Amélia Maria. Pedagogia da pesquisa-ação. Educação e pesquisa, v. 31, n. 3, 2005, p. 483-502

GoER, Charis; KÖLlER, Katharina (Hg.). Fachdidaktik Deutsch. Grundzüge der Sprach- und Literaturdidaktik. 2., überarbeitete und aktualisierte Auflage. Paderborn: Wilhelm Fink, 2016.

HERZOG, Walter. Reflexive Praktika in der Lehrerinnen- und Lehrerbildung. In: Beiträge zur Lehrerinnen- und Lehrerbildung, v. 13, n. 3, 1995, p. 253-273. Disponível em: <https://core.ac.uk/download/pdf/83642597.pdf> Acesso em 31.07. 2018.

KÖNIGS, Frank G. Sprachlehrforschung. In: SURKAMP, Carola (Hg.). Metzler Lexikon Fremdsprachendidaktik. Stuttgart: J. B. Metzler, 2017, p. 322-324.

KÜHN, Peter. Wortschatz und Wortschatzdidaktik: Tradition und Neuansätze. In: OOMENWelKe, Ingelore; AHRENHOLZ, Bernt (Hg.). Deutsch als Fremdsprache. Baltmannsweiler: Schneider Verlag Hohengehren, 2013, p. 153-164.

MoHR, Imke; SCHART, Michael. Praxiserkundungsprojekte und ihre Wirksamkeit in der Lehrerfort- und Weiterbildung. In: LEGUTKE, Michael; SCHART, Michael (Hg.). Fremdsprachendidaktische Professionsforschung: Brennpunkt Lehrerbildung. Tübingen: Narr, 2016, p. 291-322.

NEVELING, Christiane. Wortschatz und Wortschatzvermittlung. In: SURKAMP, Carola (Hg.). Metzler Lexikon Fremdsprachendidaktik. Stuttgart: J. B. Metzler, 2017, p. 378-381.

PIBID no Brasil: O que é o PIBID? Disponível em <www.pibid.pr1.ufrj.br/index.php/2012-1217-16-28-24/pibid-no-brasil> Acesso em 31.07.2018.

PUPP SPINASSÉ, Karen. Deutsch als Fremdsprache in Brasilien. Frankfurt: Lang, 2005.

Pandaemonium, São Paulo, v. 22, n. 36, jan.-abr. 2019, p. 178-206 
SANTOS, Y.; KLEINBING, H.; SCRIBELK, L.; VOERKEL, P. - Platz zum Denken!?

RICHTER, Claudia. Das Bildungswesen in Brasilien. In: ADICK, Christel (Hg.). Bildungsentwicklungen und Schulsysteme in Afrika, Asien, Lateinamerika und der Karibik. Münster: Waxmann, 2013, p. 171-189.

RIEMER, Claudia. Spracherwerb und Spracherwerbstheorien. In: SURKAMP, Carola (Hg.). Metzler Lexikon Fremdsprachendidaktik. Stuttgart: J. B. Metzler, 2017, p. 318-321.

SCHART, Michael; LEGUTKE, Michael. Lehrkompetenz und Unterrichtsgestaltung. München: Klett-Langenscheidt, 2012.

STANKE, Roberta. Cultura e interculturalidade na formação do professor de alemão no Rio de Janeiro. Unveröffentlichte Dissertation. Rio de Janeiro: UFRJ, 2014.

STANKE, Roberta; Bolacio, Ebal. Germanistik und DaF an der Universidade do Estado do Rio de Janeiro. In: DAAD (Hg.). Germanistik in Brasilien. Herausforderungen, Vermittlungswege, Übersetzungen. Göttingen: Wallstein, 2014, p. 127-129.

STORK, Antje. Wortschatzerwerb. In: HALlET, Wolfgang; KÖNIGS, Frank G. (Hg.): Handbuch Fremdsprachendidaktik. Seelze-Velber: Klett Kallmeyer, 2010, p. 104-107.

UnIVERSIDAde FEDERAL DO RIO DE JANEIRO. Catálogo CLAC. Descritivo acerca dos Cursos de Línguas Abertos à Comunidade vigentes em 2017-2018. Rio de Janeiro: UFRJ, 2017, p. 9-17. Disponível em: <https://drive.google.com/file/d/0B23bxlwjXLNabkotUlhLMkh5cFk/view> Acesso em 31.07.2018.

VOERKEL, Paul. Deutsch als Chance: Ausbildung, Qualifikation und Verbleib von Absolventen brasilianischer Deutschstudiengänge. Tese de doutorado. Jena: Friedrich-SchillerUniversität, 2017a. Disponível em: <https://www.dbthueringen.de/receive/dbt_mods_00033644> Acesso em 08.05.2018.

. Professores de alemão no Brasil - entre mitos e realidade. In: EMMEL, Ina; FRIESEN Blume, Rosvitha; HeIdermanN, Werner Ludger (Org.). Anais do $2^{\circ}$ Congresso da Associação Brasileira de Estudos Germanísticos. Florianópolis, 2017b, p. 306-314.

[im Druck]. Os cursos de Letras-Alemão no Brasil: dados, perfis, oportunidades e desafios. In: UPHOFF, Dörthe et al. (Org.). Alemão em contexto universitário: ensino, pesquisa e extensão. São Paulo: Humanitas.

WALlaCE, Michael. Action Research for Language Teachers. 14th printing. Cambridge: Cambridge University Press, 2010.

ZIERER, Klaus. Hattie für gestresste Lehrer. Kernbotschaften und Handlungsempfehlungen aus John Hatties , Visible Learning “ und „,Visible Learning for teachers “. Baltmannsweiler: Schneider Verlag Hohengehren, 2016.

ZYDATISS, Wolfgang. 22 Thesen zur Reform der universitären Fremdsprachenlehrerausbildung. Disponível em: <www.fapf.de/html/dokumente/ vdfdocs/11.htm> Acesso em 07.08.2018.

ZYNGIER, Sonia; LiBERALI, Fernanda Coelho. Caderno de reflexões para os orientadores e monitores do CLAC. Rio de Janeiro: Serviço de Publicações / FL, 2000. 
SANTOS, Y.; KLEINBING, H.; SCRIBELK, L.; VOERKEL, P. - Platz zum Denken!?

ANHANG 1

UNIVERSIDADE FEDERAL DO RIO DE JANEIRO

FACULDADE DE LETRAS / UFRJ

CURSOS DE LÍNGUAS ABERTOS À COMUNIDADE - CLAC

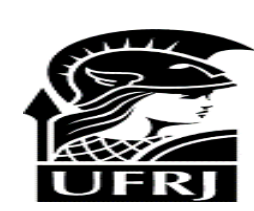

Kursleiter/in:

Klasse:

Datum: 2017

Questionário sobre as atividades de vocabulário em sala

Fragebogen zu den Aktivitäten der Wortschatzarbeit im Unterricht

Atividade 1 (Explicação com mímicas, gestos, desenhos, imagens)

a) Como você avalia a compreensão do vocabulário aprendido utilizando esse método?
(1) Muito fácil
(2) Fácil
(3) Médio
(4) Difícil
(5) Muito difícil

b) Existiu alguma dificuldade em relação a esse método?

(Sim) (Não)

Caso tenha existido, diga qual foi:

\section{Atividade 2 (Tradução direta do alemão para o português)}

c) Como você avalia a compreensão do vocabulário aprendido utilizando esse método?
(1) Muito fácil
(2) Fácil
(3) Médio
(4) Difícil
(5) Muito difícil

d) Existiu alguma dificuldade em relação a esse método? (Sim) (Não)

Caso tenha existido, diga qual foi:

\section{Panorama geral sobre as duas atividades feitas em sala}
e) Qual atividade você achou mais produtiva?
(Atividade 1)
(Atividade 2)
f) Justifique sua resposta:
g) Qual atividade você gostou mais?
(Atividade 1)
(Atividade 2)
h) Justifique sua resposta: 